\title{
Synthesis and GC-MS of 6-Alkylestradiols, Possible Aromatase Reaction Products of 6-Alkylandrostenediones
}

\author{
Mitsuteru Numazawa* and Akiko Yoshrmura \\ Tohoku College of Pharmacy, 4-1 Komatsushima 4-chome, Aobaku, Sendai 981, Japan. \\ Received March 18, 1996; accepted May 8, 1996
}

\begin{abstract}
A series of $6 \alpha$ - and $6 \beta$-alkylestradiols (5 and 6) (alkyl: methyl, ethyl, $n$-propyl, $n$-pentyl, and $n$-heptyl) were synthesized as possible aromatase reaction products of 6 -alkylandrost-4-ene-3,17-diones and their $\Delta^{1}$-derivatives, potent competitive and mechanism-based inhibitors of aromatase. Treatment of 6-oxoestradiol with Grignard reagents followed by acid-catalyzed dehydration and subsequent catalytic hydrogenation over $\mathrm{Pd}-\mathrm{C}$ gave the 6-alkylestradiols (5 and 6). GC-MS (electron impact mode) of trimethylsilyl derivatives of bis-trimethylsilyl derivatives of compounds 5 and 6 revealed that the $6 \alpha$-alkyl compounds, 5 , emerged with a longer retention time compared to the corresponding 6/3-alkyl isomers, 6 , where the retention time was in proportion to the length of the 6-alkyl chain in each series. In the MS, a molecular ion $\left(\mathrm{M}^{+}\right)$peak was the base peak for all the 6-alkylestrogens, with strong and characteristic fragment ion peaks corresponding to $\mathrm{M}^{+}-131$ and at $m / z, 325$. A selected ion monitoring method using a molecular ion will be sensitive enough for analysis of the aromatization reaction of the 6-alkylandrogens.
\end{abstract}

Key words 6-alkylestradiol; synthesis; aromatase; GC-MS; analysis

Aromatase is a cytochrome P-450 (P-450 XIXA1) enzyme complex which catalyzes the conversion of androst-4-ene-3,17-dione (androstenedione) to estrone through three sequential oxygenations of the 19-methyl group. ${ }^{1)}$ Inhibitors of aromatase have recently become of interest not only in the treatment of advanced estrogendependent breast cancer $^{2)}$ but also in the elucidation of the spatial aspects of the active-site of the enzymes as well as the still unresolved mechanism of the aromatase reaction. ${ }^{3)}$

Several 6-substituted substrate analogs, 6-bromo-, ${ }^{4)}$ 6-bromoacetoxy-, ${ }^{5}$ 6 6 -fluoro-, ${ }^{\text {) }}$ and 6-hydroperoxy-7) steroids, have been synthesized to act as probes of the aromatase active site. Recently, the structure-activity relationships of 6-alkylandrostenediones as aromatase inhibitors have revealed that aromatase has a hydrophobic binding pocket with limited accessible volume in the active site in the region corresponding to the $\mathrm{C}-6$ position of the substrate. ${ }^{8)}$ In addition, $\Delta^{1}$-analogs of the 6 -alkylandrostenediones inactivate aromatase in a suicide manner, indicating that the $\Delta^{1}$-steroids could be substrates for the enzyme. ${ }^{9)}$ On the basis of these previous findings, it seems to be important to determine whether or not these 6-alkylandrostenediones with or without a double bond at $\mathrm{C}-1$ are aromatized by catalysis of aromatase. Thus, we needed authentic samples for the identification of possible aromatized products of the 6-alkylandrogens by GC-MS. A survey of the literature revealed one report of stereospecific synthesis of $6 \alpha$ - and $6 \beta$-alkylestradiols (alkyl: methyl, isopropyl, and $\left.n-\mathrm{C}_{12} \mathrm{H}_{25}\right)$ via $\mathrm{Cr}\left(\mathrm{CO}_{3}\right)$ complexes, but their physical data, except melting points, have not been described. ${ }^{10)}$ In this report, we report the synthesis and GC-MS of various $6 \alpha$ - and $6 \beta$-alkylestradiols ( 5 and 6).

Synthesis of 6-Alkylestradiols (5 and 6) The reaction of 6-oxoestradiol (1) with Grignard reagents $(\mathrm{RMgBr}$; $\mathrm{R}=$ methyl, ethyl, $n$-propyl, $n$-pentyl, and $n$-heptyl) in tetrahydrofuran (THF) on heating under reflux gave the corresponding 6-alkyl-6-ols 2 in 32-45\% yields (Chart 1). On the basis of the ${ }^{1} \mathrm{H}-\mathrm{NMR}$ spectroscopy, the products

* To whom correspondence should be addressed were found to be $c a$. 1:1 mixtures of $6 \beta$-alkyl- $6 \alpha$-ols and their $6 \alpha$-alkyl isomers in every case $(\Delta \delta$ of 18 -methyl protons with resonances at $0.75-0.79 \mathrm{ppm}$ between the two stereoisomers $=0.01 \mathrm{ppm}$ ). It has been reported that $\mathrm{CH}_{3} \mathrm{MgBr}$ reacts with $5 \alpha-6$-keto steroids which have a 19-methyl group, with a very pronounced preference for the formation of the tertiary alcohol with an equatorial methyl group, the $6 \alpha$-methyl derivatives. ${ }^{11)}$ It seems likely that a phenolic A-ring of compound $\mathbf{1}$ might decrease the steric preference for the production of compound $\mathbf{2}$. Since the mixtures could not be separated by silica gel column chromatography, the 6-ols 2 were, without further purification, subjected to dehydration with $\mathrm{HCl}$ in $95 \% \mathrm{EtOH}$ (68-86\% yield). ${ }^{1} \mathrm{H}-\mathrm{NMR}$ spectroscopy of the dehydration products showed that the reaction produced $c a .3: 1$ to 6:1 mixtures of 6-ene steroids, 3, and 6,6-methylene analogs, 4, respectively, except the reaction with the 6-methyl compound 2a in which the 6-ene compound 3a was solely produced. $\left[{ }^{1} \mathrm{H}-\mathrm{NMR} \delta: 5.75-5.76,7-\mathrm{H}(\mathrm{s})\right.$ for 3; $5.93-6.05,6^{1}-\mathrm{H}(\mathrm{m})$ for 4$]$. The mixtures obtained from the ethyl and $n$-propyl steroids $\mathbf{2 b}$ and $\mathbf{2 c}$ were separated, respectively, by reverse-phase HPLC $\left(\mathrm{C}_{18}\right.$ column, $\mathrm{MeCN}-\mathrm{H}_{2} \mathrm{O}$ ); however, the other mixtures were used without separation for the next step. Catalytic hydrogenation of the dehydrated mixtures obtained from the $n$-pentyl and $n$-heptyl steroids $\mathbf{2 d}$ and $\mathbf{2 c}$, as well as the 6-methyl-, 6-ethyl-, and 6- $n$-propyl-6-enes $\mathbf{3 a}-\mathbf{c}$ over $\mathrm{Pd}-\mathrm{C}$ under $\mathrm{H}_{2}$, gave $\mathrm{ca}$. $1: 5$ to $1: 7$ mixtures of $6 \alpha$-alkylestradiols, 5, and their 6 $\beta$-alkyl isomers, $6(58-81 \%)(\mathrm{Ta}-$ bles 1 and 2). All of the $6 \alpha$-alkyl- and $6 \beta$-alkyl steroids, 5 and $\mathbf{6}$, were isolated in a pure form using reversephase HPLC $\left(\mathrm{C}_{18}\right.$ column, MeCN-- $\left.\mathrm{H}_{2} \mathrm{O}\right)$. Catalytic hydrogenation of the 6-alkyl-6-ene steroid, 3 , and its 6,6methylene analog, $\mathbf{4}$, under the condition used seems to occur preferentially from the $\alpha$ side, ${ }^{12)}$ suggesting that the major product would be the $6 \beta$-alkyl steroid 6. Moreover, we have previously reported that the 18-methyl protons of $6 \beta$-alkylandrost-4-enes and their $\Delta^{1}$ analogs exhibit resonance at a lower field compared to those of the corresponding $6 \alpha$-alkyl analogs on their ${ }^{1} \mathrm{H}$-NMR 


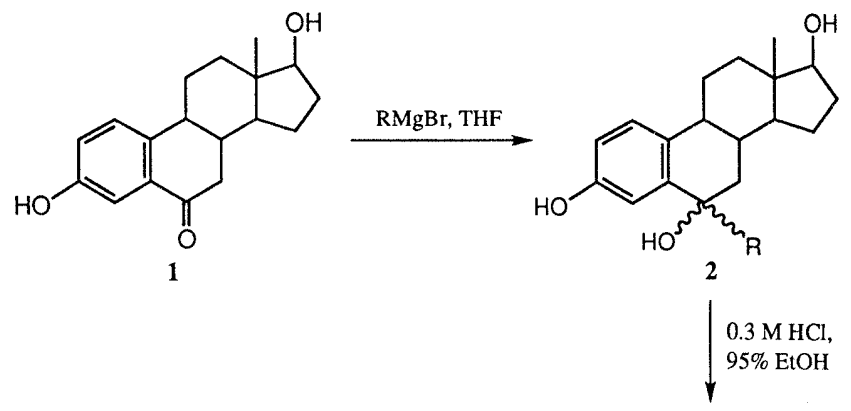

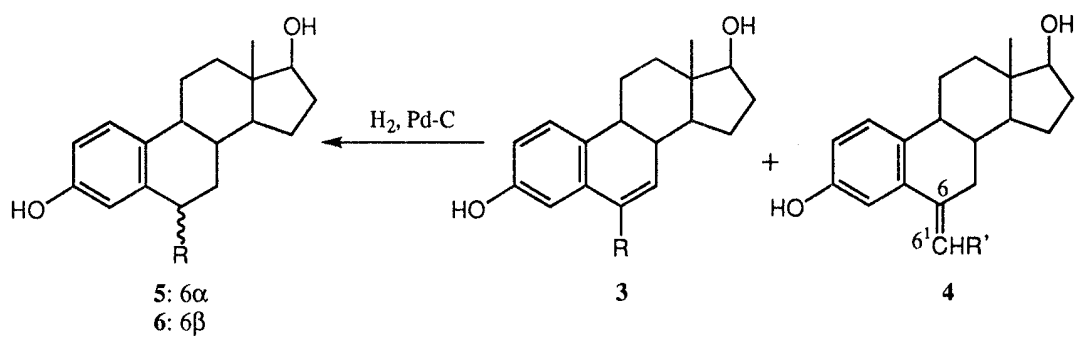

$\mathrm{R}: \mathrm{a}=\mathrm{CH}_{3} ; \quad \mathbf{b}=\mathrm{C}_{2} \mathrm{H}_{5} ; \quad \mathbf{c}=n-\mathrm{C}_{3} \mathrm{H}_{7} ; \quad \mathbf{d}=n-\mathrm{C}_{5} \mathrm{H}_{11} ; \quad \mathbf{e}=n-\mathrm{C}_{7} \mathrm{H}_{15}$

$\mathrm{R}^{\prime}: \mathbf{a}=\mathrm{CH}_{3} ; \quad \mathbf{b}=\mathrm{C}_{2} \mathrm{H}_{5} ; \mathbf{c}=n-\mathrm{C}_{4} \mathrm{H}_{9} ; \quad \mathbf{d}=n-\mathrm{C}_{6} \mathrm{H}_{13}$

Chart 1

Table 1. Physical Data for 6-Alkylestradiols 5 and 6

\begin{tabular}{|c|c|c|c|c|c|c|c|c|c|}
\hline \multirow{3}{*}{ Compound } & \multirow{3}{*}{$\mathrm{R}$} & \multirow{3}{*}{$\begin{array}{l}\text { Yield } \\
(\%)\end{array}$} & \multirow{3}{*}{$\mathrm{mp}\left({ }^{\circ} \mathrm{C}\right)$} & \multirow{3}{*}{ Purification } & \multirow{3}{*}{ Formula } & \multicolumn{4}{|c|}{ Analysis (\%) } \\
\hline & & & & & & \multicolumn{2}{|c|}{ Calculated } & \multicolumn{2}{|c|}{ Found } \\
\hline & & & & & & $\mathrm{C}$ & $\mathrm{H}$ & $\mathrm{C}$ & $\mathrm{H}$ \\
\hline \multicolumn{10}{|l|}{$6 \alpha$-Series } \\
\hline $\mathbf{5 a}$ & $\mathrm{CH}_{3}$ & 6 & Oil $(130)^{b)}$ & HPLC & $\mathrm{C}_{19} \mathrm{H}_{26} \mathrm{O}_{2}$ & \multicolumn{2}{|c|}{$286.19330^{a)}$} & \multicolumn{2}{|c|}{$286.1979^{a)}$} \\
\hline $\mathbf{5 b}$ & $\mathrm{CH}_{2} \mathrm{CH}_{3}$ & 10 & Oil & HPLC & $\mathrm{C}_{20} \mathrm{H}_{28} \mathrm{O}_{2}$ & \multicolumn{2}{|c|}{$300.20900^{a)}$} & \multicolumn{2}{|c|}{$300.2083^{a)}$} \\
\hline $5 \mathbf{c}$ & $\left(\mathrm{CH}_{2}\right)_{2} \mathrm{CH}_{3}$ & 10 & Oil & HPLC & $\mathrm{C}_{21} \mathrm{H}_{30} \mathrm{O}_{2}$ & \multicolumn{2}{|c|}{$314.22460^{a)}$} & \multicolumn{2}{|c|}{$314.2251^{a)}$} \\
\hline 5d & $\left(\mathrm{CH}_{2}\right)_{4} \mathrm{CH}_{3}$ & 11 & Oil & HPLC & $\mathrm{C}_{23} \mathrm{H}_{34} \mathrm{O}_{2}$ & \multicolumn{2}{|c|}{$342.25590^{a)}$} & \multicolumn{2}{|c|}{$342.2554^{a)}$} \\
\hline $5 e$ & $\left(\mathrm{CH}_{2}\right)_{6} \mathrm{CH}_{3}$ & 8.6 & Oil & HPLC & $\mathrm{C}_{25} \mathrm{H}_{38} \mathrm{O}_{2}$ & \multicolumn{2}{|c|}{$370.28720^{a)}$} & \multicolumn{2}{|c|}{$370.2850^{a)}$} \\
\hline $\begin{array}{c}6 \beta \text {-Series } \\
\mathbf{6 a}\end{array}$ & $\mathrm{CH}_{3}$ & 68 & $172-173(\text { oil })^{b)}$ & $\begin{array}{l}\text { Recryst. } \\
\text { (AcOEt) }\end{array}$ & $\mathrm{C}_{19} \mathrm{H}_{26} \mathrm{O}_{2}$ & 79.68 & 9.15 & 79.46 & 9.21 \\
\hline \multirow{2}{*}{$\begin{array}{l}\mathbf{6 b} \\
\mathbf{6 c}\end{array}$} & \multirow{2}{*}{$\begin{array}{l}\mathrm{CH}_{2} \mathrm{CH}_{3} \\
\left(\mathrm{CH}_{2}\right)_{2} \mathrm{CH}_{3}\end{array}$} & 48 & \multirow{2}{*}{$\begin{array}{c}\text { Oil } \\
91-93\end{array}$} & HPLC & \multirow{2}{*}{$\begin{array}{l}\mathrm{C}_{20} \mathrm{H}_{28} \mathrm{O}_{2} \\
\mathrm{C}_{21} \mathrm{H}_{30} \mathrm{O}_{2}\end{array}$} & \multicolumn{2}{|c|}{$300.20900^{a)}$} & \multicolumn{2}{|c|}{$300.2126^{a)}$} \\
\hline & & 71 & & $\begin{array}{c}\text { Recryst. } \\
\left(\mathrm{MeOH}-\mathrm{H}_{2} \mathrm{O}\right)\end{array}$ & & 80.21 & 9.62 & 80.20 & 9.75 \\
\hline \multirow{2}{*}{$\begin{array}{l}6 d \\
6 e\end{array}$} & \multirow{2}{*}{$\begin{array}{l}\left(\mathrm{CH}_{2}\right)_{4} \mathrm{CH}_{3} \\
\left(\mathrm{CH}_{2}\right)_{6} \mathrm{CH}_{3}\end{array}$} & 48 & Oil & HPLC & $\mathrm{C}_{23} \mathrm{H}_{34} \mathrm{O}_{2}$ & 342 & $90^{a)}$ & 342 & $8^{a)}$ \\
\hline & & 52 & $144-146$ & $\begin{array}{c}\text { Recryst. } \\
\text { (AcOEt-hexane) }\end{array}$ & $\mathrm{C}_{25} \mathrm{H}_{38} \mathrm{O}_{2}$ & 81.03 & 10.34 & 80.99 & 9.87 \\
\hline
\end{tabular}

spectroscopies. $^{8,9)}$ Signals of 18 -methyl protons of the major products, 6 , appeared at 0.79 or $0.80 \mathrm{ppm}$, while those of the minor ones, 5 , resonanced at 0.75 or $0.76 \mathrm{ppm}$. Taken together, it is reasonable to assume the major products, 6 , to have a $6 \beta$-configuration. Finally, the configuration of the 6-alkyl substituents were unambiguously determined on the basis of the results of the aromatization reaction with human placental microsomes; $6 \alpha$-alkyl-substituted androstenediones were converted into the corresponding compounds, 5 . In contrast, the $6 \beta$ alkylandrogens were aromatized to the corresponding compounds 6 (to be reported elsewhere).

Gas Chromatography-Mass Spectrometry of 6-Alkylestradiols (5 and 6) Bis-trimethylsilyl (TMS) derivatives of compounds 5 and $\mathbf{6}$ were then analyzed by GC (column, fused silica DB 5)-MS (EI). Retention times $\left(t_{\mathrm{R}}\right)$ of the
$6 \alpha$-alkyl steroids, 5 , were longer than those of the corresponding $6 \beta$-isomers, 6 . In every case, the two stereoisomers were separated by more than 1.3 of resolution $\left(R_{\mathrm{s}}\right)$ under the given conditions (Table 3 ). The introduction of a methyl group at C- $6 \beta$ of estradiol decreased the $t_{\mathrm{R}}$ value (14.9 vs. $15.0 \mathrm{~min})$; in contrast, the same chemical modification at C-6 $\alpha$ increased it ( 15.1 vs. $15.0 \mathrm{~min})$. The $t_{\mathrm{R}}$ value increased in proportion to the carbon number of the 6-alkylchain in both the $6 \alpha$ - and $6 \beta$-series. The results indicate that the bis-TMS derivatives of the $6 \beta$-alkyl compound having a quasi-axial conformation at C-6 are more hydrophobic and volatile than the corresponding quasi-equatorial $6 \alpha$-isomer. Further study is required to understand the structure-retention time relationship, since there is currently no data concerning the conformational analysis of the alkylsteroids. 
Table 2. Spectral Data for 6-Alkylestradiols 5 and $\mathbf{6}$

\begin{tabular}{|c|c|c|c|c|c|c|c|c|}
\hline \multirow{2}{*}{ Compd. } & \multirow{2}{*}{$\mathrm{R}$} & \multicolumn{5}{|c|}{${ }^{1} \mathrm{H}-\mathrm{NMR}\left(\mathrm{CDCl}_{3}\right), \delta$} & \multirow{2}{*}{$\begin{array}{c}\text { UV } \\
(\text { EtOH) } \\
\text { nm }(\varepsilon)\end{array}$} & \multirow{2}{*}{$\begin{array}{c}\mathrm{IR} \\
(\mathrm{KBr} \text { or neat }) \\
\mathrm{cm}^{-1}\end{array}$} \\
\hline & & $18-\mathrm{Me}$ & $6-\left(\mathrm{CH}_{2}\right)_{n} \mathrm{Me}$ & $6-\mathrm{H}$ & $1-\mathrm{H}$ & Other signals & & \\
\hline \multicolumn{9}{|c|}{$6 \alpha$-Series } \\
\hline $5 a$ & $\mathrm{CH}_{3}$ & 0.76 & $1.29(\mathrm{t}, J=6.9 \mathrm{~Hz})$ & $2.92(\mathrm{~m})$ & $7.15(\mathrm{~d}, J=8.6 \mathrm{~Hz})$ & $\begin{array}{l}6.63(\mathrm{dd}, J=8.6,2.6 \mathrm{~Hz}, 2-\mathrm{H}) \\
6.76(\mathrm{~d}, J=2.6 \mathrm{~Hz}, 4-\mathrm{H})\end{array}$ & $280.8(2160)$ & 3415,2923 \\
\hline $\mathbf{5 b}$ & $\mathrm{CH}_{2} \mathrm{CH}_{3}$ & 0.76 & $0.87(\mathrm{t}, J=7.4 \mathrm{~Hz})$ & $2.83(\mathrm{~m})$ & $7.14(\mathrm{~d}, J=8.6 \mathrm{~Hz})$ & $\begin{array}{l}6.63(\mathrm{dd}, J=8.6,2.6 \mathrm{~Hz}, 2-\mathrm{H}) \\
6.76(\mathrm{~d}, J=2.6 \mathrm{~Hz}, 4-\mathrm{H})\end{array}$ & $278.8(2160)$ & 3423,2928 \\
\hline $5 c$ & $\left(\mathrm{CH}_{2}\right)_{2} \mathrm{CH}_{3}$ & 0.75 & $0.92(\mathrm{t}, J=7.3 \mathrm{~Hz})$ & $2.85(\mathrm{~m})$ & $7.14(\mathrm{~d}, J=8.6 \mathrm{~Hz})$ & $\begin{array}{l}6.61(\mathrm{dd}, J=8.6,2.3 \mathrm{~Hz}, 2-\mathrm{H}) \\
6.75(\mathrm{~d}, J=2.3 \mathrm{~Hz}, 4-\mathrm{H})\end{array}$ & $281.2(2250)$ & 3414,2955 \\
\hline $5 d$ & $\left(\mathrm{CH}_{2}\right)_{4} \mathrm{CH}_{3}$ & 0.75 & $0.88(\mathrm{t}, J=6.6 \mathrm{~Hz})$ & $2.85(\mathrm{~m})$ & $7.14(\mathrm{~d}, J=8.6 \mathrm{~Hz})$ & $\begin{array}{l}6.62(\mathrm{dd}, J=8.6,2.3 \mathrm{~Hz}, 2-\mathrm{H}) \\
6.74(\mathrm{~d}, J=2.3 \mathrm{~Hz}, 4-\mathrm{H})\end{array}$ & $281.2(2380)$ & 3412,2926 \\
\hline $5 e$ & $\left(\mathrm{CH}_{2}\right)_{6} \mathrm{CH}_{3}$ & 0.75 & $0.88(\mathrm{t}, J=6.6 \mathrm{~Hz})$ & $2.84(\mathrm{~m})$ & $7.14(\mathrm{~d}, J=8.6 \mathrm{~Hz})$ & $\begin{array}{l}6.62(\mathrm{dd}, J=8.4,2.3 \mathrm{~Hz}, 2-\mathrm{H}) \\
6.74(\mathrm{~d}, J=2.3 \mathrm{~Hz}, 4-\mathrm{H})\end{array}$ & $280.9(2210)$ & 3418,2927 \\
\hline $\begin{array}{c}6 \beta \text {-Seri } \\
\mathbf{6 a}\end{array}$ & $\mathrm{CH}_{3}$ & 0.80 & $1.29(\mathrm{~d}, J=7.3 \mathrm{~Hz})$ & $2.92(\mathrm{~m})$ & $7.14(\mathrm{~d}, J=9.6 \mathrm{~Hz})$ & $\begin{array}{l}6.61-6.64 \\
(2 \mathrm{H}, \mathrm{m}, 2-\mathrm{H} \text { and } 4-\mathrm{H})\end{array}$ & $280.8(2100)$ & 3309,2958 \\
\hline $\mathbf{6 b}$ & $\mathrm{CH}_{2} \mathrm{CH}_{3}$ & 0.79 & $1.00(\mathrm{t}, J=7.4 \mathrm{~Hz})$ & $2.61(\mathrm{~m})$ & $7.14(\mathrm{~d}, J=9.6 \mathrm{~Hz})$ & $\begin{array}{l}6.61-6.64 \\
(2 \mathrm{H}, \mathrm{m}, 2-\mathrm{H} \text { and } 4-\mathrm{H})\end{array}$ & $280.8(2060)$ & 3424,2925 \\
\hline $6 c$ & $\left(\mathrm{CH}_{2}\right)_{2} \mathrm{CH}_{3}$ & 0.80 & $0.95(\mathrm{t}, J=7.3 \mathrm{~Hz})$ & $2.71(\mathrm{~m})$ & $7.14(\mathrm{~d}, J=9.6 \mathrm{~Hz})$ & $\begin{array}{l}6.60-6.65 \\
(2 \mathrm{H}, \mathrm{m}, 2-\mathrm{H} \text { and } 4-\mathrm{H})\end{array}$ & $281.2(2060)$ & 3402,2927 \\
\hline 6d & $\left(\mathrm{CH}_{2}\right)_{4} \mathrm{CH}_{3}$ & 0.80 & $0.91(\mathrm{t}, J=6.6 \mathrm{~Hz})$ & $2.69(\mathrm{~m})$ & $7.14(\mathrm{~d}, J=9.2 \mathrm{~Hz})$ & $\begin{array}{l}6.60-6.65 \\
(2 \mathrm{H}, \mathrm{m}, 2-\mathrm{H} \text { and } 4-\mathrm{H})\end{array}$ & $281.2(2340)$ & 3400,2927 \\
\hline $6 e$ & $\left(\mathrm{CH}_{2}\right)_{6} \mathrm{CH}_{3}$ & 0.80 & $0.90(\mathrm{t}, J=6.3 \mathrm{~Hz})$ & $2.69(\mathrm{~m})$ & $7.14(\mathrm{~d}, J=9.2 \mathrm{~Hz})$ & $\begin{array}{l}6.60-6.63 \\
(2 \mathrm{H}, \mathrm{m}, 2-\mathrm{H} \text { and } 4-\mathrm{H})\end{array}$ & $281.2(2200)$ & 3400,2926 \\
\hline
\end{tabular}

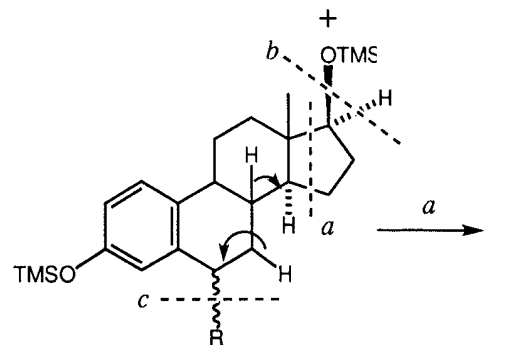<smiles>COc1ccc2c(c1)CCC=C1C[C@H](C)CCC12</smiles>

M-131<smiles>[3H][13CH3]</smiles>

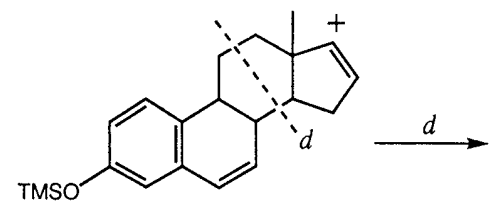

$m / z 325$

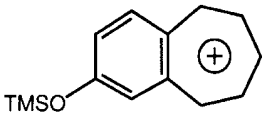

$m / z 229$

Chart 2

Electron impact mass spectra of the bis-TMS derivatives of all the 6-alkylestradiols (5 and 6) showed a molecular ion $\left(\mathrm{M}^{+}\right)$as the base peak with two characteristic ions of $\mathrm{M}^{+}-131$ and $m / z 325$ (Table 4). The fragment $\mathrm{M}^{+}-131$ can be derived from the cleavage of the C(13)-C(17) and C(14)-C(15) bonds of the bis-TMS derivatives ( $a$, Chart 2 ) followed by deprotonation at the C-8 position, as previously reported for the bisTMS derivative of estradiol. ${ }^{13)}$ The fission of $\mathrm{C}-\mathrm{H}$ and $\mathrm{C}-\mathrm{O}$ bonds at the $\mathrm{C}-17$ position $(b)$, together with a $\mathrm{C}(6)-\mathrm{C}\left(6^{1}\right)$ bond $(c)$, as well as deprotonation at the C-7 position, may produce an other ion at $\mathrm{m} / \mathrm{z} 325$
Table 3. Gas Chromatographic Retention Times for $6 \alpha-$ and $6 \beta$ Alkylestradiols 5 and $\mathbf{6}$

\begin{tabular}{lcc}
\hline \hline \multirow{2}{*}{$\mathrm{R}$} & \multicolumn{2}{c}{ Retention time, $t_{\mathrm{R}}, \min$} \\
\cline { 2 - 3 } & $\mathbf{5}(6 \boldsymbol{\alpha})$ & $\mathbf{6}(6 \beta)$ \\
\hline Estradiol, $\mathrm{H}$ & 15.0 & - \\
a, $\mathrm{CH}_{3}$ & 15.1 & 14.9 \\
b, $\mathrm{C}_{2} \mathrm{H}_{5}$ & 15.9 & 15.7 \\
c, $n-\mathrm{C}_{3} \mathrm{H}_{7}$ & 16.8 & 16.4 \\
d, $n-\mathrm{C}_{5} \mathrm{H}_{11}$ & 20.1 & 19.5 \\
$\mathbf{e}, n-\mathrm{C}_{7} \mathrm{H}_{15}$ & 24.5 & 23.5 \\
\hline
\end{tabular}

Table 4. Fragmentation of 6-Alkylestradiols (5 and 6) by Electron Impact Mass Spectrometry

\begin{tabular}{|c|c|c|c|}
\hline \multirow{2}{*}{ Compounds } & \multicolumn{3}{|c|}{ Fragment ion, $m / z$ (relative abundance) $)^{a)}$} \\
\hline & $\mathrm{M}^{+}$ & $\mathrm{M}^{+}-131$ & Others \\
\hline $6 \alpha$-Methyl $\mathbf{5 a}$ & $430(100)$ & $299(100)$ & $129(28), 73(60)$ \\
\hline $6 \beta$-Methyl $\mathbf{6 a}$ & $430(100)$ & $299(43)$ & $129(10), 73(10)$ \\
\hline $6 \alpha$-Ethyl $\mathbf{5 b}$ & $444(100)$ & $313(28)$ & $325(23), 207(28)$ \\
\hline $6 \beta$-Ethyl $\mathbf{6 b}$ & $444(100)$ & $313(19)$ & $229(11), 73(10)$ \\
\hline $6 \alpha-n$-Propyl $5 c$ & $458(100)$ & $327(66)$ & $325(68), 207(31), 73(70)$ \\
\hline $6 \beta-n$-Propyl $\mathbf{6 c}$ & $458(100)$ & $327(66)$ & $325(68), 229(28), 73(68)$ \\
\hline $6 \alpha-n$-Pentyl 5d & $486(100)$ & $355(30)$ & $207(35), 147(42), 73(43)$ \\
\hline $6 \beta$-n-Pentyl 6d & $486(100)$ & $355(45)$ & $207(26), 73(32)$ \\
\hline $6 \alpha-n$-Heptyl 5e & $5148100)$ & 383 ( 29$)$ & $325(51), 73(53)$ \\
\hline $6 \beta$-n-Heptyl 6e & $514(100)$ & 383 ( 29) & $325(41), 73(59)$ \\
\hline
\end{tabular}

a) Fragment ions with more than $10 \%$ of relative intensity to the base peak were listed.

which, when further fragmented, causes the rupture of the vinylogous benzylic $\mathrm{C}(8)-\mathrm{C}(14)$ bond, followed by a second benzylic cleavage to furnish the siloxybenztropylium ion $(m / z 229) .{ }^{14)}$ Using a selected ion monitoring (SIM) method with the $\mathrm{M}^{+}$, we could quantitate the TMS 
derivatives as low as $50 \mathrm{pg}$, respectively $(S / N>10)$. This sensitivity is sufficient for the detection of the aromatization reaction of the 6-alkylandrogens. Study of the aromatization reaction using this GC-MS method is now underway in our laboratory.

\section{Experimental}

Melting points were measured on a Yanagimoto melting point apparatus and are uncorrected. IR spectra were recorded on a Perkin-Elmer FT-IR 1725X spectrophotometer and UV spectra in $95 \%$ EtOH solution on a Hitachi 150-20 spectrophotometer. ${ }^{1} \mathrm{H}-\mathrm{NMR}$ spectra were obtained in $\mathrm{CDCl}_{3}$ solution with JEOL EX $270(270 \mathrm{MHz})$ spectrometers using tetramethylsilane as an internal standard, and MS (HR-MS) with a JEOL JMS-DX 303 spectrometer. GC-MS was carried out with a Finnigan MAT SSQ GC-MS instrument. TLC was performed on E. Merck pre-coated silica gel plates. Column chromatography was conducted with silica gel (E. Merck, 70-230 mesh). HPLC was carried out using a Waters Model 510 pump, YMC Pack-D-ODS- 5 column $(250 \mathrm{~mm} \times 20 \mathrm{~mm}$ i.d.), and a UV detector $(280 \mathrm{~nm})$. Grignard reagents in THF solutions were purchased from Aldrich Chemical Co. Bis(trimethylsilyl)trifluoroacetamide (BSTFA) was obtained from Tokyo Kasei Kogyo Co. 6-Oxoestradiol was synthesized according to the method previously reported by Akanni and Marples. ${ }^{15)}$

Grignard Reactions of 6-Oxoestradiol (1) 30 molar eq. of Grignard reagent $(\mathrm{RMgBr}: \mathrm{R}=$ methyl, ethyl, $n$-propyl, $n$-pentyl, or $n$-heptyl) in THF $(68 \mathrm{ml})$ was added to a solution of compound $1(2.3 \mathrm{mmol})$ in THF $(16 \mathrm{ml})$, and the mixture was heated under reflux for about $30 \mathrm{~h}$ in an $\mathrm{N}_{2}$ stream. After the solution was cooled, saturated $\mathrm{NH}_{4} \mathrm{Cl}$ solution $(200 \mathrm{ml})$ was added and the product was extracted with AcOEt $(250 \mathrm{ml} \times 2)$. The combined organic layer was washed with saturated $\mathrm{NaHCO}_{3}$ solution and water to neutrality, dried with $\mathrm{Na}_{2} \mathrm{SO}_{4}$, and evaporated to dryness leaving a residue which was purified by column chromatography (hexane-AcOEt) to give 6-alkylestra-1,3,5(10)-triene$3,6,17 \beta$-triol (2) consisting of two $6 \alpha$ - and $6 \beta$-alkyl stereoisomers as a solid or oil (yield: methyl, $45 \%$; ethyl, $43 \%$; $n$-propyl, $38 \%$; $n$-pentyl, $32 \%$; $n$-heptyl, $35 \%)$. The isomers could not be separated by HPLC, therefore, compound 2 was used without further purification for the next step reaction.

Dehydration of the 6-Alkyl-6-ols 2 with $\mathbf{H C l} 0.3 \mathrm{M} \mathrm{HCl}(2.3 \mathrm{ml}$, $0.7 \mathrm{mmol})$ was added to a stirred solution of compound $2(0.94 \mathrm{mmol})$ in $95 \% \mathrm{EtOH}(20 \mathrm{ml})$ and the mixture was stirred at room temperature for $24 \mathrm{~h}$. After this time, $0.1 \mathrm{M} \mathrm{NaOH}$ solution $(6 \mathrm{ml})$ was added to the mixture and the solvent was concentrated under reduced pressure, diluted with AcOEt $(100 \mathrm{ml})$, washed with water to neutrality, and dried with $\mathrm{Na}_{2} \mathrm{SO}_{4}$. Evaporation of the solvent gave an oily product which was purified by column chromatography to afford a mixture of 6-alkylestra-1,3,5(10),6-tetraene-3,17 $\beta$-diol (3) and 6,6-alkylmethyleneestra-1,3,5(10)-triene-3,17 $\beta$-diol (4), except the reaction of the 6 -methyl compound $2 \mathrm{a}$, which gave only the 6-ene steroid $\mathbf{3 a}$ (yield: methyl, $68 \%$; ethyl, $71 \%$; $n$-propyl, $66 \% ; n$-pentyl, $75 \% ; n$-heptyl, $86 \%$ ). The mixtures obtained from the 6-ethyl and 6-n-propyl steroid $\mathbf{2 b}$ and $\mathbf{2 c}$ were respectively separated by reverse-phase HPLC [solvent, $\mathrm{MeCN}-\mathrm{H}_{2} \mathrm{O}$ $(60: 40, v / v), 5 \mathrm{ml} / \mathrm{min}$ for the separation of $\mathbf{2 b}$ and $\mathrm{MeCN}-\mathrm{H}_{2} \mathrm{O}(65: 35$, $\mathrm{v} / \mathrm{v}$ ), and $5 \mathrm{ml} / \mathrm{min}$ for the separation of $2 \mathrm{c}] .{ }^{1} \mathrm{H}-\mathrm{NMR}$ spectrum of the mixture produced from the 6-n-pentyl derivative $2 \mathrm{~d}$ revealed it to be a 6:1 mixture of the 6-ene 3d and the 6,6-methylene analog 4c [3d: $\delta 0.77$ (s, 18-Me), $3.76(\mathrm{t}, J=8.4 \mathrm{~Hz}, 17 \alpha-\mathrm{H}), 5.76(\mathrm{~s}, 7-\mathrm{H}), 6.67$ (dd, $J=2.6$ $8.3 \mathrm{~Hz}, 2-\mathrm{H}), 6.79(\mathrm{~d}, J=2.6 \mathrm{~Hz}, 4-\mathrm{H}), 7.11(\mathrm{~d}, J=8.3 \mathrm{~Hz}, 1-\mathrm{H}) ; 4 \mathrm{c}: \delta$ $0.76(\mathrm{~s}, 18-\mathrm{Me}), 3.74(\mathrm{t}, J=8.4 \mathrm{~Hz}, 17 \alpha-\mathrm{H}), 5.94\left(\mathrm{~m}, \mathrm{C}=\mathrm{CHCH}_{2}-\right), 6.67$ (dd, $J=2.6,8.3 \mathrm{~Hz}, 2-\mathrm{H}), 7.03(\mathrm{~d}, J=2.6 \mathrm{~Hz}, 4-\mathrm{H}), 7.14(\mathrm{~d}, J=8.3 \mathrm{~Hz}$, $1-\mathrm{H})]$, and the spectrum of the mixture from the 6-n-heptyl compound, $2 \mathrm{e}$, revealed it to be a $3: 1$ mixture of the 6-ene $3 \mathrm{e}$ and the 6,6-methylene analog 4d [3e: $\delta 0.77(\mathrm{~s}, 18-\mathrm{Me}), 3.76(\mathrm{t}, J=8.4 \mathrm{~Hz}, 17 \alpha-\mathrm{H}), 5.75(\mathrm{~s}, 7-\mathrm{H})$, $6.67(\mathrm{dd}, J=2.6,8.3 \mathrm{~Hz}, 2-\mathrm{H}), 6.78(\mathrm{~d}, J=2.6 \mathrm{~Hz}, 4-\mathrm{H}), 7.12(\mathrm{~d}, J=8.3 \mathrm{~Hz}$, $1-\mathrm{H}) ; 4 \mathrm{~d}: \delta 0.76(\mathrm{~s}, 18-\mathrm{Me}), 3.74(\mathrm{t}, J=8.4 \mathrm{~Hz}, 17 \alpha-\mathrm{H}), 5.95(\mathrm{~m}$, $\left.\mathrm{C}=\mathrm{CH}^{\mathrm{HCH}}{ }_{2}-\right), 6.67(\mathrm{dd}, J=2.6,8.1 \mathrm{~Hz}, 2-\mathrm{H}), 7.03(\mathrm{~d}, J=2.4 \mathrm{~Hz}, 4-\mathrm{H})$, $7.15(\bar{d}, J=8.1 \mathrm{~Hz}, 1-\mathrm{H})]$. The other mixtures were used, without further purification, for the next step reaction.

6-Methylestra-1,3,5(10),6-tetraene-3,17 $\beta$-diol (3a) Yield: $68 \%$ (oil). ${ }^{1} \mathrm{H}-\mathrm{NMR} \delta: 0.77(3 \mathrm{H}, \mathrm{s}, 18-\mathrm{Me}), 3.75(1 \mathrm{H}, \mathrm{t}, J=8.4 \mathrm{~Hz}, 17 \alpha-\mathrm{H}), 5.78$ $(1 \mathrm{H}, \mathrm{s}, 7-\mathrm{H}), 6.68(1 \mathrm{H}, \mathrm{dd}, J=2.6,8.3 \mathrm{~Hz}, 2-\mathrm{H}), 6.77(1 \mathrm{H}, \mathrm{d}, J=2.6 \mathrm{~Hz}$, 4-H), $7.12(1 \mathrm{H}, \mathrm{d}, J=8.3 \mathrm{~Hz}, 1-\mathrm{H})$. FT-IR (neat): $3352,2938(\mathrm{OH}) \mathrm{cm}^{-1}$. UV $\lambda_{\max }: 261(\varepsilon=8400), 300(\varepsilon=4000) \mathrm{nm}$. HR-MS $m / z$ : Calcd for
$\mathrm{C}_{19} \mathrm{H}_{24} \mathrm{O}_{2}\left(\mathrm{M}^{+}\right)$, 284.17760; found, 284.1777.

6-Ethylestra-1,3,5(10),6-tetraene-3,17 $\boldsymbol{\beta}$-diol (3b) Yield: $58 \%$ (oil). HPLC: $t_{\mathrm{R}}=30.6 \mathrm{~min} .{ }^{1} \mathrm{H}-\mathrm{NMR} \delta: 0.77(3 \mathrm{H}, \mathrm{s}, 18-\mathrm{Me}), 1.13(3 \mathrm{H}, \mathrm{t}$, $\left.J=7.4 \mathrm{~Hz}, 6-\mathrm{CH}_{2} \mathrm{Me}\right), 5.76(1 \mathrm{H}, \mathrm{s}, 7-\mathrm{H}), 6.67(1 \mathrm{H}, \mathrm{dd}, J=2.6,8.3 \mathrm{~Hz}$, 2-H), $6.80(\mathrm{lH}, \mathrm{d}, J=2.6 \mathrm{~Hz}, 4-\mathrm{H}), 7.13(1 \mathrm{H}, \mathrm{d}, J=8.3 \mathrm{~Hz}, 1-\mathrm{H})$. FT-IR (neat): $3414,2936(\mathrm{OH}) \mathrm{cm}^{-1}$. UV $\lambda_{\text {max }}: 261(\varepsilon=7200), 303(\varepsilon=3000)$ nm. HR-MS $m / z$ : Calcd for $\mathrm{C}_{20} \mathrm{H}_{26} \mathrm{O}_{2}\left(\mathrm{M}^{+}\right), 298.19330$; found, 298.1920 .

6-n-Propylestra-1,3,5(10),6-tetraene-3,17 $\boldsymbol{\beta}$-diol (3c) Yield: $53 \%$. mp $160-162^{\circ} \mathrm{C}$. HPLC: $t_{\mathrm{R}}=32.0 \mathrm{~min} .{ }^{1} \mathrm{H}-\mathrm{NMR} \delta: 0.77(3 \mathrm{H}, \mathrm{s}, 18-\mathrm{Me}), 0.93$ $\left(3 \mathrm{H}, \mathrm{t}, J=7.3 \mathrm{~Hz}, 6-\mathrm{CH}_{2} \mathrm{CH}_{2} \mathrm{Me}\right), 3.76(1 \mathrm{H}, \mathrm{t}, J=8.4 \mathrm{~Hz}, 17 \alpha-\mathrm{H}), 5.76$ $(1 \mathrm{H}, \mathrm{s}, 7-\mathrm{H}), 6.67(1 \mathrm{H}, \mathrm{dd}, J=2.6,8.3 \mathrm{~Hz}, 2-\mathrm{H}), 6.79(1 \mathrm{H}, \mathrm{d}, J=2.6 \mathrm{~Hz}$, 4-H), $7.13(1 \mathrm{H}, \mathrm{d}, J=8.3 \mathrm{~Hz}, 1-\mathrm{H})$. FT-IR $(\mathrm{KBr}): 3405,2955(\mathrm{OH}) \mathrm{cm}^{-1}$. UV $\lambda_{\text {max }}: 262(\varepsilon=6900), 304(\varepsilon=2700) \mathrm{nm}$. Anal. Calcd for $\mathrm{C}_{21} \mathrm{H}_{28} \mathrm{O}_{2}$ : C, $80.73 ; \mathrm{H}, 9.03$. Found: C, $80.79 ; \mathrm{H}, 8.86$.

6,6-Ethyleneestra-1,3,5(10)-triene-3,17 $\beta$-diol (4a) Yield: 13\% (oil). HPLC: $t_{\mathrm{R}}=28.9 \mathrm{~min} .{ }^{1} \mathrm{H}-\mathrm{NMR} \delta: 0.76(3 \mathrm{H}, \mathrm{s}, 18-\mathrm{Me}), 1.76(3 \mathrm{H}, \mathrm{d}$, $J=6.9 \mathrm{~Hz}, \mathrm{C}=\mathrm{CHMe}), 3.74(1 \mathrm{H}, \mathrm{t}, J=8.4 \mathrm{~Hz}, 17 \alpha-\mathrm{H}), 6.05(1 \mathrm{H}, \mathrm{m}$, $\mathrm{C}=\mathrm{C} \underline{\mathrm{HMe}}), 6.67(1 \mathrm{H}, \mathrm{dd}, J=2.6,8.5 \mathrm{~Hz}, 2-\mathrm{H}), 7.02(1 \mathrm{H}, \mathrm{d}, J=2.6 \mathrm{~Hz}$, 4-H), 7.15 (1 H, d, $J=8.5 \mathrm{~Hz}, 1-\mathrm{H})$. FT-IR (neat): $3400,2980(\mathrm{OH}) \mathrm{cm}^{-1}$. UV $\lambda_{\text {max }}: 260(\varepsilon=10500), 302(\varepsilon=4300) \mathrm{nm}$. HR-MS $m / z$ : Calcd for $\mathrm{C}_{20} \mathrm{H}_{26} \mathrm{O}_{2}\left(\mathrm{M}^{+}\right)$, 298.19330; found, 298.1922.

6,6-n-Propyleneestra-1,3,5(10)-triene-3,17 $\beta$-diol (4b) Yield: $13 \%$. mp $112-115^{\circ} \mathrm{C}$. HPLC: $t_{\mathrm{R}}=30.5 \mathrm{~min} .{ }^{1} \mathrm{H}-\mathrm{NMR} \delta: 0.76(3 \mathrm{H}, \mathrm{s}, 18-\mathrm{Me}), 1.06$ $\left(3 \mathrm{H}, \mathrm{t}, J=7.6 \mathrm{~Hz}, 6-\mathrm{CHCH}_{2} \mathrm{Me}\right), 3.74(1 \mathrm{H}, \mathrm{t}, J=8.2 \mathrm{~Hz}, 17 \alpha-\mathrm{H}), 5.76$ $(1 \mathrm{H}, \mathrm{s}, 7-\mathrm{H}), 6.67(1 \mathrm{H}, \mathrm{dd}, J=2.6,8.3 \mathrm{~Hz}, 2-\mathrm{H}), 5.94(1 \mathrm{H}, \mathrm{dd}, J=5.4$, $\left.8.1 \mathrm{~Hz}, \mathrm{C}=\mathrm{CHCH}_{2} \mathrm{Me}\right), 7.05(1 \mathrm{H}, \mathrm{d}, J=2.6 \mathrm{~Hz}, 4-\mathrm{H}), 7.14(1 \mathrm{H}, \mathrm{d}$, $J=8.3 \mathrm{~Hz}, 1-\mathrm{H})$. FT-IR $(\mathrm{KBr}): 3403,2960(\mathrm{OH}) \mathrm{cm}^{-1}$. UV $\lambda_{\text {max }}: 259$ $(\varepsilon=10800), 303(\varepsilon=4600) \mathrm{nm}$. Anal. Calcd for $\mathrm{C}_{21} \mathrm{H}_{28} \mathrm{O}_{2}: \mathrm{C}, 80.77 ; \mathrm{H}$, 8.97. Found: C, $80.75 ; \mathrm{H}, 9.11$.

Catalytic Hydrogenation of the 6-Ene Steroids $3 \quad 5 \% \mathrm{Pd}-\mathrm{C}(80 \mathrm{mg})$ was added separately to a solution of the 6 -ene steroids $3 \mathbf{a}-\mathbf{c}$ or the mixtures of the 6-ene steroids $3 \mathrm{~d}$ and $3 \mathrm{e}$ and the 6,6-methylene derivatives $4 \mathbf{c}$ and $4 \mathbf{d}(c a .0 .54 \mathrm{mmol})$ in dry EtOH $(15 \mathrm{ml})$. The mixture was vigorously stirred under $\mathrm{H}_{2}$ for 2 to $3 \mathrm{~h}$. The catalyst was removed by filtration and the filtrate was evaporated to give a mixture of $6 \alpha$ - and $6 \beta$-alkyl steroids, 5 and $\mathbf{6}$, which was purified by column chromatography (hexane-AcOEt) followed by reverse-phase HPLC. Conditions: MeCN$\mathrm{H}_{2} \mathrm{O}(50: 50, \mathrm{v} / \mathrm{v}), 5 \mathrm{ml} / \mathrm{min}$ for $5 \mathrm{a}\left(t_{\mathrm{R}}=44.3 \mathrm{~min}\right)$ and $\mathbf{6 a}\left(t_{\mathrm{R}}=41.4 \mathrm{~min}\right)$; $\mathrm{MeCN}-\mathrm{H}_{2} \mathrm{O}(70: 30, \mathrm{v} / \mathrm{v}), 5 \mathrm{ml} / \mathrm{min}$ for $5 \mathbf{b}\left(t_{\mathrm{R}}=23.0 \mathrm{~min}\right)$ and $\mathbf{6 b}$ $\left(t_{\mathrm{R}}=21.4 \mathrm{~min}\right)$ and for $5 \mathbf{c}\left(t_{\mathrm{R}}=29.0 \mathrm{~min}\right)$ and $\mathbf{6 c}\left(t_{\mathrm{R}}=26.5 \mathrm{~min}\right)$; $\mathrm{MeCN}-\mathrm{H}_{2} \mathrm{O}(80: 20, \mathrm{v} / \mathrm{v}), 5 \mathrm{ml} / \mathrm{min}$ for $5 \mathrm{~d}\left(t_{\mathrm{R}}=32.0 \mathrm{~min}\right)$ and $\mathbf{6 d}$ $\left(t_{\mathrm{R}}=29.2 \mathrm{~min}\right)$ and $7 \mathrm{ml} / \mathrm{min}$ for $5 \mathrm{e}\left(t_{\mathrm{R}}=42.2 \mathrm{~min}\right)$ and $6 \mathbf{e}\left(t_{\mathrm{R}}=35.2 \mathrm{~min}\right)$.

GC-MS A Finnigan MAT SSQ GC-MS instrument was used. Gas chromatographic conditions: column, $30 \mathrm{~m} \times 0.250 \mathrm{~mm}$ i.d. fused silica DB5 (J \& W Scientific, CA, U.S.A.); column temperature, from $50^{\circ} \mathrm{C}$ at $25^{\circ} \mathrm{C} / \mathrm{min}$ to $250^{\circ} \mathrm{C}$ and then at $10^{\circ} \mathrm{C} / \mathrm{min}$ to $280^{\circ} \mathrm{C}$; carrier gas, He at a flow rate of $60 \mathrm{ml} / \mathrm{min}$. Mass spectrometric conditions: ionization energy, $70 \mathrm{eV}$; ion source temperature, $150^{\circ} \mathrm{C}$.

Derivatization of the 6-Alkylestradiols 5 and 6 with BSTFA BSTFA $(30 \mu \mathrm{l})$ was added separately to a solution of the estradiols 5 and $\mathbf{6}$ in dry pyridine $(30 \mu \mathrm{l})$. The mixture was heated at $60^{\circ} \mathrm{C}$ for $30 \mathrm{~min}$ and then the solvent was removed under a stream of $\mathrm{N}_{2}$. The residue was dissolved in anhydrous hexane $(25 \mu \mathrm{l})$, and $2 \mu \mathrm{l}$ portions of the solution were subjected to analysis.

Acknowledgments We thank Dr. K. Yamashita of Nihon Kayaku Co., Ltd. for his useful suggestion regarding GC-MS analysis.

\section{References}

1) Thompson E. A., Jr., Siiteri P. K., J. Biol. Chem., 249, 5373-5378 (1974); Kellis J., Jr., Vickery L. E., ibid., 262, 4413-4420 (1987); Yoshida N., Osawa Y., Biochemistry, 30, 3003-3010 (1991).

2) For example see: Harvey H. A., Lipton A., Santen R. J., Cancer Res. (Suppl.), 42, 3261s-3269s (1982); Brodie A. M. H., Coombes R. C., Dowsett M., J. Steroid Biochem., 27, 899-903 (1987); Manni A., J. Cell Biochem., 17G, 242-246 (1993); Johnston S. R. D., Smith I. E., Doody D., Jacobs S., Robertshaw H., Dowsett M., Cancer Res., 54, 5875-5881 (1994).

3) For example see: Covey D. F., "Steroid Biosynthesis Inhibitors: Pharmaceutical and Agrochemical Aspects," ed. by Berg D., Plemel M., Ellis Horwood Ltd., Chichester, England, 1988, pp. $534-571$; Banting L., Nicholls P. J., Shaw M. A., Smith H. J., "Progress in Medicinal Chemistry," ed. by Ellis G. P., West G. B., Elsevier Science Publishers, B. W., Amsterdam, 1989, Vol. 26, pp. 253-298. 
4) Osawa Y., Osawa Y., Coon M. J., Endocrinology, 121, 1010-1016 (1987)

5) Numazawa M., Tsuji M., Osawa Y., Steroids, 48, 347-357 (1986); Numazawa M., Tsuji M., Mutsumi A., Nagaoka M., Chem. Pharm. Bull., 37, 735-737 (1989).

6) Mann J., Pietrzak B., J. Chem. Soc., Perkin Trans. I, 1983, $2681-2685$

7) Tan L., Hrycay F. G., Matsumoto K., J. Steroid Biochem., 19, 1329-1338 (1983); Tan L., Peffit A., Biochem. Biophys. Res. Commun., 128, 613-620 (1985).

8) Numazawa M., Oshibe M., J. Med. Chem., 37, 1312-1319 (1994) idem, Steroids, 60, 576-581 (1995).

9) Numazawa M., Oshibe M., Yamaguchi S., Tachibana M., J. Med Chem., 39, 1033-1038 (1996).
10) Top S., Vessieres A., Abjean J.-P., Jaouen G., J. Chem. Soc., Chem. Commun., 1984, 428-429.

11) Fieser L. F., Rigaudy J., J. Am. Chem. Soc., 73, 4660-4662 (1951); Davis M., Summer G. H. R., J. Chem. Soc., 1960, 4707-4708.

12) Augustive R. L., "Organic Reactions in Steroid Chemistry," ed. by Fried J., Edwards J. A., Van Nostrand Reinhold Co., New York, 1972, pp. $111-144$.

13) Miyazaki H., Ishibashi M., Itoh M., J. Chromatogr., 135, 109-116 (1977).

14) Budzikiewicz H., Djerassi H., Williams D. H., "Structure Elucidation of Natural Products by Mass Spectrometry," Holden-Day, Inc., San Francisco, 1964, p. 59.

15) Akanni A., Marples B. A., Synth. Commun., 14, 713-715 (1984). 